\title{
Incidence of constitutive and inducible clindamycin resistance among hospital-associated Staphylococcus aureus
}

\author{
B. Sasirekha $\cdot$ M. S. Usha $\cdot$ J. A. Amruta $\cdot$ \\ S. Ankit $\cdot$ N. Brinda $\cdot$ R. Divya
}

Received: 28 December 2012/Accepted: 19 March 2013/Published online: 2 April 2013

(C) The Author(s) 2013. This article is published with open access at Springerlink.com

\begin{abstract}
Clindamycin is one of the important alternative antibiotics in the therapy of Staphylococcus aureus infections. Clinical failure of clindamycin therapy has been reported due to multiple mechanisms that confer resistance to macrolides, lincosamides and Streptogramin B $\left(\mathrm{MLS}_{\mathrm{B}}\right)$ antibiotics. In vitro routine tests for clindamycin susceptibility may fail to detect inducible clindamycin resistance due to erm genes, resulting in the treatment failure. Although data from the developed countries have shown to be enormity of the problem, data from the developing countries are lacking. The aim of the study was to distinguish different resistance phenotypes in erythromycinresistant $S$. aureus by a simple double-disc diffusion test (D test). A total of $153 \mathrm{~S}$. aureus isolates were subjected to routine antibiotic susceptibility testing, including cefoxitin disc $(30 \mu \mathrm{g})$ and by oxacillin screen agar. Inducible clindamycin resistance was tested by ' $D$ test' as per CLSI guidelines. Odds ratios (OR) and $95 \%$ confidence intervals $(95 \% \mathrm{CI})$ were calculated. $P$ values were calculated using SPSS (version 18). Among 153 S. aureus isolates, 42 $(27.45 \%)$ were resistant to methicillin, whereas 111 $(72.54 \%)$ were methicillin susceptible. Out of the 63 $(41.17 \%)$ erythromycin-resistant $S$. aureus isolates, 14 $(9.15 \%)$ showed inducible resistance $[P=0.0002$, odds ratio (OR) $18.30 ; 95 \%$ confidence interval (CI) 8.72-25.77), 20 (13.07\%)] showed constitutive resistance $(P=0.002$, OR $14.38,95 \%$ CI 5.33-21.49), while the remaining $29(18.95 \%)$ showed inducible phenotype.
\end{abstract}

B. Sasirekha $(\square) \cdot$ M. S. Usha · J. A. Amruta · S. Ankit .

N. Brinda $\cdot$ R. Divya

Department of Microbiology, Center for Post Graduate Studies,

Jain University, 18/3, 9th Main, Jayanagar 3rd Block,

Bangalore 560011, India

e-mail: nagsrani@yahoo.co.in
Inducible and constitutive resistance was found to be higher in MSSA when compared with MRSA. Clinical laboratories should perform D test routinely to guide the clinicians about the inducible clindamycin resistance and to prevent misuse of antibiotics.

Keywords Inducible clindamycin $\cdot \mathrm{D}$ test . Staphylococcus aureus - Inducible $\mathrm{MLS}_{\mathrm{B}}$ phenotype

\section{Introduction}

Staphylococcus aureus (S. aureus) is a leading cause of nosocomial and community-acquired infections in every region of world. The increasing prevalence of methicillin resistance among Staphylococci is an increasing problem (Yilmaz et al. 2007). This has led to the renewed interest in the usage of macrolide-lincosamide-streptogramin B $\left(\mathrm{MLS}_{\mathrm{B}}\right)$ antibiotics to treat $S$. aureus infections with clindamycin being the preferred agent due to its excellent pharmacokinetic properties (Deotale et al. 2010). However, the wide spread use of $\mathrm{MLS}_{\mathrm{B}}$ antibiotics has led to an increase in the number of Staphylococcal strains acquiring resistance to $\mathrm{MLS}_{\mathrm{B}}$ antibiotics (Ajantha et al. 2008).

Resistance to $\mathrm{MLS}_{\mathrm{B}}$ can occur by two different mechanisms: an active efflux mechanism encoded by the $m s r A$ gene (macrolides Streptogramin B resistance) and ribosomal target modification encoded by the erm gene $\left(\mathrm{MLS}_{\mathrm{B}}\right.$ resistance) (Leclercq 2002). The expression of the $M_{L} S_{B}$ phenotype can be constitutive or inducible in the presence of low levels of inducers, such as erythromycin. erm genes encode enzymes that confer inducible or constitutive resistance to MLS agents via methylation of the $23 \mathrm{~S}$ ribosomal RNA, thereby reducing binding by MLS agents to the ribosome (Fiebelkorn et al. 2003). In vitro, S. aureus

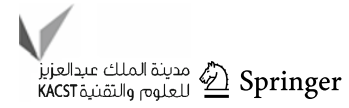


isolates with constitutive resistance are resistant to erythromycin and clindamycin, and isolates with inducible resistance are resistant to erythromycin, but appear susceptible to clindamycin. In vivo, therapy with clindamycin may select for constitutive erm mutants (Leclercq 2002) that may lead to clinical failure (Siberry et al. 2003).

$\mathrm{iMLS}_{\mathrm{B}}$ (inducible clindamycin) is not recognized using standard susceptibility test methods, including standard broth-based or agar dilution susceptibility tests (Fiebelkorn et al. 2003), including the Vitek system (Schreckenberger et al. 2004) and disc diffusion testing with erythromycin (E) and clindamycin (CL) discs (double discs) in nonadjacent positions (Gadepalli et al. 2006). Further reports on inducible clindamycin resistance are scanty in India. Therefore the present study was undertaken to determine the incidence of $M S_{B}$ resistance in the clinical isolates of $S$. aureus and to study the antibiotic sensitivity pattern of $S$. aureus isolates having the $\mathrm{iMLS}_{\mathrm{B}}$ phenotype.

\section{Materials and methods}

This prospective study was conducted for a period of 7 months from July 2010 to January 2011. A total of 153 Staphylococcal isolates were recovered from various clinical samples at the Microbiology Laboratory of Sri Bhagawan Mahaveer Jain hospital, a tertiary care hospital in Bangalore. Duplicate isolates from the same patient were not included in the study. Of $153 \mathrm{~S}$. aureus isolates, 110 $(71.89 \%)$ were recovered from pus, $13(8.49 \%)$ from sputum, $11(7.18 \%)$ from ear swab, $9(5.88 \%)$ from blood, $5(3.26 \%)$ from urine and $5(3.26 \%)$ from tissue bits.

Isolates were identified up to species level by conventional methods (Gram stain, growth on mannitol salt agar, slide and tube coagulase test, DNase test and by biochemical test) (Kloos and Banerman 1999).

\section{Antibiotic susceptibility testing}

The isolates were subjected to susceptibility testing by Kirby Bauer disc diffusion method on Mueller-Hinton agar plates using erythromycin $(15 \mu \mathrm{g})$, clindamycin $(2 \mu \mathrm{g})$, mupirocin $(5 \mu \mathrm{g})$, fusidic acid $(30 \mu \mathrm{g})$, pristinomycin $(15 \mu \mathrm{g})$, linezolid $(30 \mu \mathrm{g})$, vancomycin $(30 \mu \mathrm{g})$, teicoplanin $(30 \mu \mathrm{g})$, rifampicin $(5 \mu \mathrm{g})$, chloramphenicol $(30 \mu \mathrm{g})$, co-trimoxazole $(30 \mu \mathrm{g})$, ciprofloxacin $(5 \mu \mathrm{g})$, gentamicin $(30 \mu \mathrm{g})$, amikacin $(30 \mu \mathrm{g})$, and tetracycline $(30 \mu \mathrm{g})$. The results were interpreted as per Clinical Laboratory Standards Institute (CLSI) guidelines (Clinical and Laboratory Standards Institute 2009) methicillin resistance was detected by cefoxitin disc diffusion method and by oxacillin screen agar $(5 \% \mathrm{NaCl}, 6 \mu \mathrm{g} / \mathrm{ml}$ oxacillin).
D-Test

Isolates that were erythromycin resistant was tested for inducible resistance by the 'D test' as per CLSI guidelines. Erythromycin $(15 \mu \mathrm{g})$ disc was placed at a distance of $15 \mathrm{~mm}$ (edge to edge) from clindamycin $(2 \mu \mathrm{g})$ on Mueller-Hinton agar plates previously inoculated with 0.5 McFarland bacterial suspensions. Plates were analyzed after $18 \mathrm{~h}$ of incubation at $37{ }^{\circ} \mathrm{C}$. Interpretation of the inhibition zone diameters was as follows: If an isolate was erythromycin resistant and clindamycin susceptible, with a D-shaped inhibition zone around the clindamycin disc, it was considered to be positive for inducible resistance (D test positive, iMLS $S_{B}$ phenotype). If the isolate was erythromycin resistant and clindamycin susceptible, with both zones of inhibition showing a circular shape, the isolate was considered to be negative for inducible resistance (D test negative, MS phenotype), but to have an active efflux pump. If the isolate was erythromycin resistant and clindamycin resistant, the isolate was considered to have the macrolide-lincosamide-Streptogramin B constitutive ( cMLS $_{\mathrm{B}}$ phenotype) (Steward et al. 2005). The quality control of the erythromycin and clindamycin disc was performed with $S$. aureus ATCC 25923.

Statistical analysis

Demographic data were collected and SPSS version 18 was used for all statistical analysis. Odds ratios (OR) and $95 \%$ confidence intervals $(95 \% \mathrm{CI})$ were calculated. $P<0.05$ was considered statistically significant.

\section{Results}

Of the $153 \mathrm{~S}$. aureus isolates, $42(27.45 \%)$ were resistant to methicillin-resistant $S$. aureus (MRSA) and 111 $(72.54 \%)$ were methicillin-susceptible $S$. aureus (MSSA). Of the total $153 \mathrm{~S}$. aureus isolates, 144 isolates $(94.11 \%)$ were coagulase positive and $9(5.88 \%)$ were coagulase negative. A total of 38 of the coagulase-positive $S$. aureus isolates showed methicillin resistant and four coagulasenegative isolates showed methicillin resistance.

Of the total 42 MRSA, 32 (76.19\%) isolates belonged to male and $10(23.80 \%)$ to female patients. Our study showed the highest percentage of MRSA occurrence in patients with the age group of 20-30 years. The number of MRSA isolates was significantly different among various age groups $(P<0.0001)$.

Out of the 153 S. aureus isolates, $63(41.17 \%)$ of them were erythromycin resistant. These isolates when subjected to D test, $20(13.07 \%)$ isolates showed resistant to erythromycin and clindamycin indicating constitutive $\mathrm{MLS}_{\mathrm{B}}$ 
phenotype. Out of the 43 isolates that showed clindamycin sensitivity, $14(9.15 \%)$ isolates showed positive D test indicating inducible $\mathrm{MLS}_{\mathrm{B}}$ phenotype, while 29 (18.95\%) showed true sensitivity to clindamycin (D test negative indicating MS phenotype). 58.82\% had the susceptible phenotype (E-S, CL-S) (Table 1; Fig. 1).

Constitutive MLS $_{\mathrm{B}}$ phenotype was $5.22 \%$ and the inducible $\mathrm{MLS}_{\mathrm{B}}$ phenotype was $0.65 \%$ in MRSA, while in methicillin sensitive Staphylococcal isolates, the constitutive MLS $_{\mathrm{B}}$ phenotype was $7.84 \%$ and the inducible MLS $_{\mathrm{B}}$ phenotype was $8.49 \%$.

The E-S and CL-S phenotype predominated over the inducible resistance phenotype and constitutive resistance phenotype among MRSA and MSSA isolates. The percentage of inducible and constitutive resistance was higher amongst MSSA isolates when compared with MRSA isolates. Out of the $14 \mathrm{iMLS}_{\mathrm{B}}$ phenotype $S$. aureus isolates, 11 $(78.57 \%)$ isolates were isolated from pus, followed by 2 $(14.28 \%)$ isolates that were isolated from ear swab and 1 $(7.14 \%)$ isolate from tissue bits. iMLS $_{\mathrm{B}}$ phenotype was found more in males $(71.42 \%)$.

When the results were statistically compared between methicillin-sensitive Staphylococcal isolates, the constitutive CL-R phenotype was determined to be 1.5 times greater $(P=0.002$, OR $14.38,95 \%$ CI $5.33-21.49)$ and the inducible resistance phenotype 13 times greater $(P=0.0002$, OR $18.30,95 \%$ CI $8.72-25.77)$ than that in methicillin-resistant Staphylococcal isolates.

The susceptibility of $i M L S_{B}$ phenotypes isolated were amikacin $85.71 \%$, gentamicin $78.57 \%$, ciprofloxacin $50 \%$, cotrimoxazole $57.14 \%$ and tetracycline $85.71 \%$. $\mathrm{iMLS}_{\text {В }}$ phenotype $S$. aureus isolates were $100 \%$ sensitive to vancomycin, teicoplanin, mupirocin, fusidic acid and linezolid, respectively.

Table 1 Susceptibility to erythromycin and clindamycin among all Staphylococcal isolates

\begin{tabular}{llll}
\hline Phenotypes & $\begin{array}{l}\text { MRSA } \\
(\%)\end{array}$ & $\begin{array}{l}\text { MSSA } \\
(\%)\end{array}$ & $\begin{array}{l}\text { Total } \\
(\%)\end{array}$ \\
\hline $\begin{array}{l}\text { E-S, CL-S } \\
\left.\begin{array}{l}\text { E-R, CL-R (constitutive } \\
\text { MLS }\end{array}\right)\end{array}$ & $\begin{array}{c}26(16.99) \\
8(5.22)\end{array}$ & $\begin{array}{l}64(41.83) \\
12(7.84)\end{array}$ & $\begin{array}{l}90(58.82) \\
\text { B }\end{array}$ \\
$\left.\begin{array}{l}\text { E-R, CL-S, D test } \\
\text { positive (inducible } \\
\text { MLS }\end{array}\right)$ & $1(0.65)$ & $13(8.49)$ & $14(9.15)$ \\
$\begin{array}{l}\text { E-R, CL-S, D test } \\
\text { negative (MS) }\end{array}$ & $9(5.88)$ & $20(13.07)$ & $29(18.95)$ \\
\begin{tabular}{l} 
Total \\
\hline
\end{tabular} & $42(28.74)$ & $111(71.23)$ & 153 \\
\hline
\end{tabular}

$E$ erythromycin, $C L$ clindamycin, $S$ sensitive, $R$ resistant, constitutive $M L S_{B}$ constitutive resistance to clindamycin, inducible $M L S_{B}$ inducible resistance to clindamycin, $M S$ ms phenotype

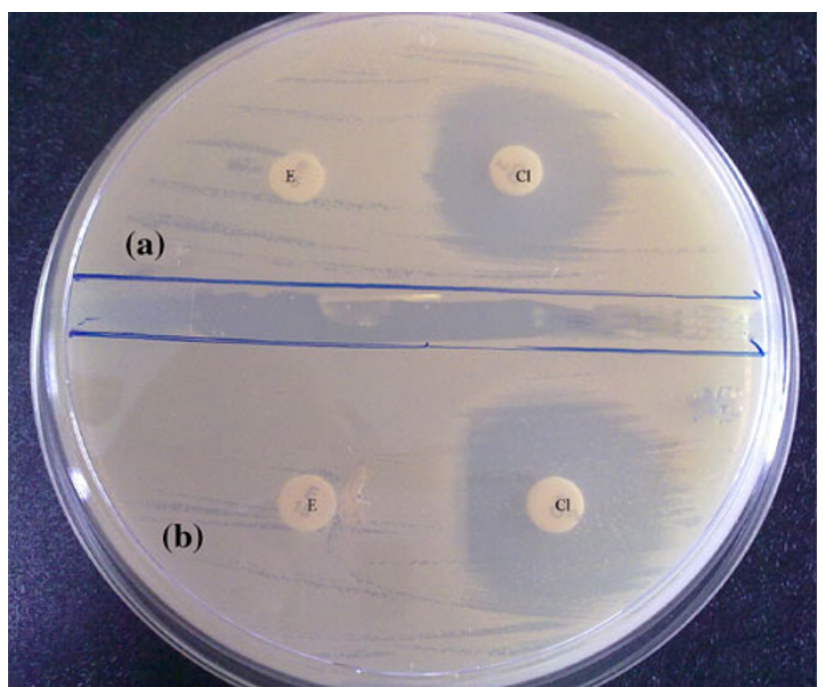

Fig. 1 Disc diffusion test for inducible clindamycin resistance. a Circular zone of inhibition around clindamycin suggestive of MS phenotype. b D-shaped zone of inhibition around clindamycin suggestive of inducible MLS $_{\mathrm{B}}$ phenotype

\section{Discussion}

The increasing frequency of Staphylococcal infections among patients and changing patterns in antimicrobial resistance have led to renewed interest in the use of clindamycin therapy to treat such infections (Frank et al. 2002). Clindamycin is frequently used to treat skin and bone infections because of its tolerability, cost, oral form and excellent tissue penetration, and the fact that it accumulates in abscesses and no renal dosing adjustments are needed (Kasten 1999). Good oral absorption makes it an important option in outpatient therapy or as follow-up after intravenous therapy. Clindamycin is a good alternative for the treatment of both methicillin-resistant and susceptible Staphylococcal infections (Fiebelkorn et al. 2003).

The very high rates of methicillin resistance among S. aureus isolates have been noted in developed countries; especially, in Western Pacific regions both in communityacquired and nosocomial infections (Diekema et al. 2001). In West Asia, MRSA prevalence ranged from 12 to $49.4 \%$ in six different hospitals of Saudi Arabia (Hussain et al. 2008; Baddour et al. 2006). In European countries, MRSA rates varied from $0.6 \%$ in Sweden to $40.2-45 \%$ in Belgium, Greece, Ireland, Italy, UK, and Israel (Blandino et al. 2004; Sader et al. 2006). In a study performed in 17 different regions of Russia, methicillin resistance among S. aureus strains was between 0 and $89.5 \%$. In our study, methicillin resistance $S$. aureus was found to be $27.45 \%$. Similar prevalence rate of MRSA was obtained from other workers in India-22.8 \% by Pal and Ayyagari (1991), $26.9 \%$ by Shittu and Lin (2006) and $26.6 \%$ Mehta et al. (2007). Although lesser and higher percentage was 
obtained by other workers-2.4\% Pulimood et al. (1996), 54.85 by Dar et al. (2006) and $65 \%$ by Borg et al. (2006). The differences in the prevalence of MRSA among different countries and between different regions in a country could be due to difference in the study design, population and geographical distribution and the variation is probably due to differential clonal expansion and drug pressure in community. Further, it emphasizes the importance of local surveillance in generating relevant local resistance data that can guide empiric therapy.

In our study, there was no isolate with reduced susceptibility to glycopeptides, and all isolates were found susceptible to vancomycin, teicoplanin, fusidic acid, mupirocin and linezolid. Rahbar and Hajia (2007) also found all iMLS $S_{B}$ isolates susceptible to linezolid and vancomycin. Disc diffusion test for vancomycin in Staphylococci is no longer recommended by CLSI starting from 2009 and that the ability of teicoplanin disc diffusion test to differentiate resistant from susceptible strains is not known (Clinical and Laboratory Standards Institute 2009).

The incidence of $M L S_{B}$ resistance varies significantly by geographical region. In our study, the percentage of inducible resistance and MS phenotype was higher amongst MSSA (8.49 and $13.07 \%$ ) when compared with MRSA (0.65 and $5.88 \%)$. This was in concordance with a few of the studies reported before. Schreckenberger et al. (2004) and Levin et al. (2005) showed higher percentage of inducible resitance in MSSA (19-20\%) as compared to MRSA (7-12\%), $12.5 \%$ MRSA and $68 \%$ MSSA, respectively. The true incidence of the MLS $_{\mathrm{B}}$ phenotype of $S$. aureus depends on the patient population studied, and the geographical region, the hospital characteristics and methicillin susceptibility (MRSA or MSSA).

In our study, $8.49 \%$ MSSA isolates were of the iMLS $_{B}$ phenotype, which is in concordance with other workers, who have found that 4-15\% of their MSSA isolates were of the iMLS B $_{\text {B }}$ henotype (Yilmaz et al. 2007). O'Sullivan et al. (2006) reported that a $15-\mathrm{mm}$ distance, in an edge to edge position, had a $100 \%$ sensitivity and specificity, while the 22-mm distance, in an edge to edge position, had a sensitivity of $87 \%$ and a specificity of $100 \%$ when compared with the presence of the erm gene as the gold standard for the detection of inducible CL resistance. Further Ajantha et al. (2008) in their study identified additional $14 \%$ iMLS $_{B}$ strains with $15 \mathrm{~mm}$ edge to edge interdisc distance. In the present study, we have maintained the narrow disc range to reduce the error rate of identifying iMLS $\mathrm{B}_{\mathrm{B}}$ isolates.

In the present study, $13.07 \%$ of erythromycin-resistant Staphylococcal isolates showed true clindamycin susceptibility. Patients with infections caused by such isolates can be treated with clindamycin without emergence of resistance during therapy. In the current study, $\mathrm{iMLS}_{\mathrm{B}}$ phenotype was found more in males $(71.42 \%)$. Male patient predominance most likely due to the fact that exposure is greater.

In the light of the restricted range of antibiotics available for the treatment of methicillin-resistant Staphylococcal infections and the known limitations of vancomycin, clindamycin should be considered for the management of serious soft tissue infections. Further, using clindamycin use of vancomycin can be avoided (Gupta et al. 2009). In addition, such testing can provide information about resistant to MLS phenotype group of antibiotics and can be useful for surveillance studies related to MLS resistance in Staphylococci.

Acknowledgments We gratefully acknowledge Mrs. Geetha Bhat, Consultant Microbiologist, Sri Bhagawan Mahaveer Jain hospital for providing clinical specimens.

\section{Conflict of interest None.}

Open Access This article is distributed under the terms of the Creative Commons Attribution License which permits any use, distribution, and reproduction in any medium, provided the original author(s) and the source are credited.

\section{References}

Ajantha GS, Kulkarni RD, Shetty J, Shubhada C, Jain P (2008) Phenotypic detection of inducible clindamycin resistance among Staphylococcus aureus isolates by using the lower limit of recommended inter-disk distance. Indian J Pathol Microbiol $51: 376-378$

Baddour MM, Abuelkheir MM, Fatani AJ (2006) Trends in antibiotic susceptibility patterns and epidemiology of MRSA isolates from several hospitals in Riyadh, Saudi Arabia. Ann Clin Microbiol Antimicrob 5:30

Blandino G, Marchese A, Ardito F, Fadda G, Fontana R, Lo Cascio G et al (2004) Antimicrobial susceptibility profiles of Pseudomonas aeruginosa and Staphylococcus aureus isolated in Italy from patients with hospital-acquired infections. Int $\mathrm{J}$ Antimicrob Agents 24:515-518

Borg M, Scicluna E, De Kraker M, Van de Sande-Bruinsma N, Tiemersma E, Gur D et al (2006) Antibiotic resistance in the southeastern Mediterrenean-preliminary results from the AR medical project. European surveillance 11(7):639

Clinical and Laboratory Standards Institute (2009) Performance standards for antimicrobial susceptibility testing; nineteenth informational supplement M100-S19. CLSI, Wayne

Dar JA, Thoker MA, Khan JA, Ali A, Khan MA, Rizwan M et al (2006) Molecular epidemiology of clinical and carrier strains of methicillin resistant Staphylococcus aureus in the hospital settings of north India. Ann Clin Microbiol Antimicrobial 5(1):22

Deotale V, Mendiratta DK, Raut U, Narang P (2010) Inducible clindamycin resistance in Staphylococcus aureus isolated from clinical samples. Indian J Med Microbiol 28:124-126

Diekema DJ, Pfaller MA, Schmitz FJ, Smayevsky J, Bell J, Jones RN et al (2001) Survey of infections due to Staphylococcus species: frequency of occurrence and antimicrobial susceptibility of isolates collected in the United States, Canada, Latin America, Europe, and the western pacific region for the SENTRY Antimicrobial Surveillance Program, 1997-1999. Clin Infect Dis 32(2):114-132 
Fiebelkorn KR, Crawford SA, McElmeel ML, Jorgensen JH (2003) Practical disc diffusion method for detection of inducible clindamycin resistance in Staphylococcus aureus and coagulase-negative Staphylococci. J Clin Microbiol 41:4740-4744

Frank AL, Marcinak JF, Mangat PD, Tjhio JT, Kelkar S, Schreckenberger PC et al (2002) Clindamycin treatment of methicillinresistant Staphylococcus aureus infections in children. Pediatr Infect Dis J 21:530-534

Gadepalli R, Dhawan B, Mohanty S, Kapil A, Das BK, Chaudhry R (2006) Inducible clindamycin resistance in clinical isolates of Staphylococcus aureus. Indian J Med Res 123:571-573

Gupta V, Datta P, Rani H, Chander J (2009) Inducible clindamycin resistance in Staphylococcus aureus: a study from north India. J Postgrad Med 55:176-179

Hussain W, Ikram A, Butt T, Faraz A, Hussain A, Wiqar MA (2008) In vitro susceptibility pattern of Staphylococcus aureus clinical isolates at Rawalpindi. Pak J Pathol 19(1):12-15

Kasten MJ (1999) Clindamycin, metronidazole, and chloramphenicol. Mayo Clin Proc 74:825-833

Kloos WE, Banerman TL (1999) Staphylococcus and micrococcus. In: Murray PR, Baron EJ, Pfaller MA, Tenover FC, Yolken RH (eds) Manual of clinical microbiology, 7th edn. ASM Press, Washington, DC, pp 264-282

Leclercq R (2002) Mechanisms of resistance to macrolides and lincosamides: nature of the resistance elements and their clinical implications. Clin Infect Dis 34:482-492

Levin TP, Suh B, Axelrod P, Truant AL, Fekete T (2005) Potential clindamycin resistance in clindamycin-susceptible, erythromycin-resistant Staphylococcus aureus: report of a clinical failure. Antimicrob Agents Chemother 49:1222-1224

Mehta M, Dutta P, Gupta V (2007) Bacterial isolates from burn wound infections and their antibiograms: an eight-year study. Indian J Plast Surg 40:25-28

O'Sullivan MV, Cai Y, Kong F, Zeng X, Gilbert GL (2006) Influence of disk separation distance on accuracy of the disk approximation test for detection of inducible clindamycin resistance in Staphylococcus spp. J Clin Microbiol 44:4072-4076
Pal N, Ayyagari A (1991) Drug resistance pattern of methicillin resistant Staphylococcus aureus. Ind Paediatrics 28:731-733

Pulimood TB, Lalitha MK, Jesudson MV, Pandian R, Selwyn JJ (1996) The spectrum of antimicrobial resistance among methicillin resistant Staphylococcus aureus in a tertiary care centre in India. Ind J Med Res 103:212-215

Rahbar M, Hajia M (2007) Inducible clindamycin resistance in Staphylococcus aureus: a cross-sectional report. Pak J Biol Sci 10:189-192

Sader HS, Streit JM, Fritsche TR, Jones RN (2006) Antimicrobial susceptibility of gram-positive bacteria isolated from European medical centers: results of the Daptomycin Surveillance Programme (2002-2004). Clin Microbiol Infect 12:844-852

Schreckenberger PC, Ilendo E, Ristow KL (2004) Incidence of constitutive and inducible resistance in Staphylococcus aureus and coagulase-negative Staphylococci in a community and a tertiary care hospital. J Clin Microbiol 42:2777-2779

Shittu AO, Lin J (2006) Antimicrobial susceptibility patterns and characterization of clinical isolates of Staphylococcus aureus in KwaZulu-Natal province, South Africa. BMC Infect Dis 6:125

Siberry GK, Tekle T, Carroll K, Dick J (2003) Failure of clindamycin treatment of methicillin-resistant Staphylococcus aureus expressing inducible clindamycin resistance in vitro. Clin Infect Dis 37:1257-1260

Steward CD, Raney PM, Morrell AK, Williams PP, McDougal LK, Jevitt L et al (2005) Testing for induction of clindamycin resistance in erythromycin-resistant isolates of Staphylococcus aureus. J Clin Microbiol 43:1716-1721

Yilmaz G, Aydin K, Iskender S, Caylan R, Koksal I (2007) Detection and prevalence of inducible clindamycin resistance in Staphylococci. J Med Microbiol 56:342-345 